\title{
Experimental Investigation of Fluid Mixing Process with Newly Designed Conduit-Type Mixer and Fin-Type Mixer*
}

\author{
Md. Tauhedul Azam¹, Donghyuk Kang1\#, Hiroyuki Hirahara1, Kazuhisa Murata², \\ Yasufumi Shimoji ${ }^{3}$ \\ ${ }^{1}$ Graduate School of Science and Engineering, Saitama University, Saitama, Japan \\ ${ }^{2}$ Eddy Plus Corp., Saitama, Japan \\ ${ }^{3}$ Aquatechs Corp., Saitama, Japan \\ Email: "dhkang@mail.saitama-u.ac.jp
}

How to cite this paper: Azam, Md.T., Kang, D., Hirahara, H, Murata, K. and Shimoji, Y. (2019) Experimental Investigation of Fluid Mixing Process with Newly Designed Conduit-Type Mixer and Fin-Type Mixer. Journal of Flow Control, Measurement \& Visualization, 7, 101-110. https://doi.org/10.4236/jfcmv.2019.72008

Received: December 25, 2018

Accepted: March 6, 2019

Published: April 4, 2019

Copyright $\odot 2019$ by author(s) and Scientific Research Publishing Inc. This work is licensed under the Creative Commons Attribution International License (CC BY 4.0).

http://creativecommons.org/licenses/by/4.0/

\begin{abstract}
In the present paper, fluid mixing process in a stirred tank with a newly designed conduit-type mixer has been investigated by the flow visualization and the concentration measurement. The results of the new conduit-type mixer are compared with a conventional fin-type mixer. Although the concentration measurement indicates that the mixing time by the conduit-type mixer is slightly longer than that by the fin-type mixer, the flow visualization shows that the conduit-type mixer generates the wider circulatory flow pattern and stirs the fluid over a vast area of the tank. Consequently, it is indicated that the conduit-type mixer has an advantage to stir the fluid uniformly in space.
\end{abstract}

\section{Keywords}

Rotating Jet, Flow Pattern, Flow Visualization, Concentration Measurement

\section{Introduction}

Many kinds of mixers have been utilized in a variety of mixing process over a wide area. For example, in chemical, pharmaceutical, food and paint industries, a lot of kinds of mixers are adopted to distinct purposes, e.g. blending of miscible liquids, contacting of immiscible liquids, emulsification process, mixing of multiphase systems, etc. The scope of mixing operations in academic and perspective of industrial research has been reported by Paul et al. [1], Harnby et al. [2], Oldshue [3], Uhl and Gray [4], Cheremisinoff [5]. They provided the de${ }^{\star}$ A part of this work was presented at 15th International Conference Fluid Dynamics (ICFD15) in Sendai, 7-9 Nov. 2018. 
tailed information about the mixing operations and discussed the reasons about poor mixing. The major reasons of poor mixing are the inappropriate selection of the mixing equipment such as mixer, mixing tank, baffles, draft tube, etc. and their scale-up techniques, lack of the understanding of the mixing mechanism of the mixer etc.

For excellent mixing achievement, it is necessary to improve the mixing mechanism of the mixer to ensure the mixing quality and efficiency. The flow pattern in the mixer is important to interpret the mixing mechanism of the mixer and depends on the geometry of the mixer and tank. In order to identify the flow pattern in stirred tank, flow visualization experiment has been conducted by using tracers and detector such as color tracer and acid-base with indicator (e.g. Rodriguez et al. [6], Norwood and Metzner [7], Lamberto et al. [8]), chemical reaction such as reaction between sodium-thiosulfate and iodine (e.g. Hiraoka et al. [9]), and particle with laser illumination (Metzner and Taylor [10]).

The present paper investigates a newly designed rotating jet mixer (C_mix, TM by Eddy plus Corp.), which is referred as conduit-type mixer as shown in Figure 1(a). This mixer has L-shaped channel as shown in Figure 1(b). When the conduit-type mixer rotates, fluid is sucked at the bottom inlet and ejected from side outlet radially as a round jet due to the centrifugal force (see Figure $1(b))$. Since it is supposed that the conduit type has various advantages such as low shear stress and safety relative to the conventional fin type, this will be applied to the wide field.

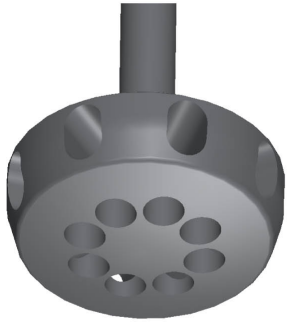

(a)

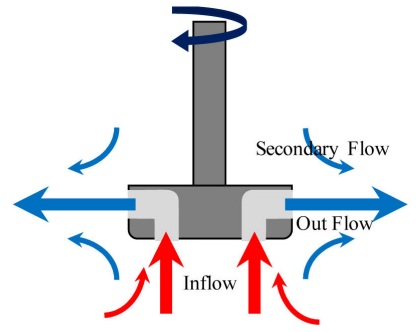

(b)

Figure 1. Schematic view of test mixer. (a) Conduit-type mixer; (b) Mixing mechanism of the conduit-type mixer.

The objective of this paper is to inspect the flow pattern of a conduit-type mixer with 8 channels by the experimental techniques. The flow pattern of the conduit-type mixer was identified by the flow visualization with particle tracer technique. The local concentration rate was measured by using image intensity of dyed water. The results of the present conduit-type mixer were compared with a conventional fin-type mixer with 3 blades.

\section{Experimental Equipment and Method}

\subsection{Visualization Experiment}

The schematic diagram of the experimental setup for visualization is presented 
in Figure 2. The experiment was carried out in a cylindrical vessel of $D=0.6 \mathrm{~m}$ diameter. The tank was filled with water of height equal to the tank diameter, $H$ $=D$. The conduit-type and fin-type mixers with the diameter, $d=D / 10$ were tested. The mixer position, $h$ was changed as shown in Table 1 . The mixer was supported and driven with a shaft of $d / 5$ diameter as shown in Figure 2. Here, the top is open to the air.

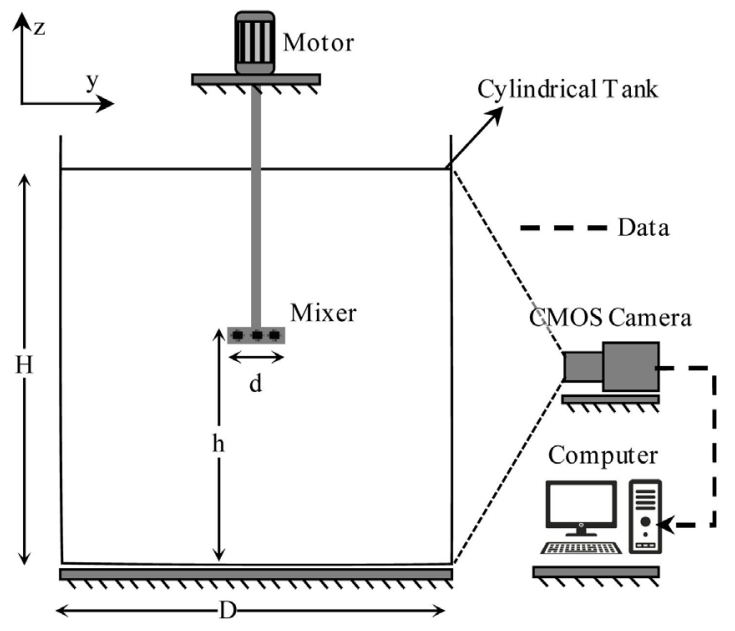

Figure 2. Schematic diagram of the visualization experimental setup.

Table 1. Experimental conditions of flow visualization.

\begin{tabular}{lll}
\hline Mixer type & $:$ & Conduit-type, Fin-type \\
\hline Mixer position, $h$ & $:$ & $6 / 8 H, 5 / 8 H, 4 / 8 H, 3 / 8 H, 2 / 8 H$ \\
\hline Rotation rate, $n$ & $:$ & $\begin{array}{l}150 \mathrm{rpm}(\mathrm{Re}=282,700), 250 \mathrm{rpm}(\mathrm{Re}=471,200), \\
350 \mathrm{rpm}(\mathrm{Re}=659,700), 450 \mathrm{rpm}(\mathrm{Re}=848,200)\end{array}$ \\
\hline Time, $t$ & $:$ & $00^{\prime} 00^{\prime}-5^{\prime} 00^{\prime \prime}, 10^{\prime} 00^{\prime \prime}-10^{\prime} 30^{\prime \prime}, 15^{\prime} 00^{\prime \prime}-15^{\prime} 30^{\prime \prime}$ \\
\hline
\end{tabular}

As a tracer, DIAION HP20 of diameter $250 \mu \mathrm{m}( \pm 10 \%)$ and density $1.01 \mathrm{~g} / \mathrm{ml}$ was used in flow visualization. The specific gravity and loading ratio of the particle were $1.01 \%$ and $0.015 \%$ in weight, so the influence of the buoyancy and mass was negligible in the experiment.

A CMOS camera (IDT M5) with $2288 \times 1728$ pixels in resolution, 8 bit in depth was used for sequential image capturing. The light source is a LED (IDT, LED-8) of luminous flux $3700 \mathrm{~lm}$. The image sequence was captured at 8 frame rates per second for time $t=00^{\prime} 00^{\prime \prime}-5^{\prime} 00^{\prime \prime}$ and 20 frame rates per second for other conditions.

The experiment was performed under the 120 conditions for the combination of mixer positions $h$, rotation rates $n$, and duration time $t$ as described in Table 1. The Reynolds number, $R e$ for the stirred tank is defined by

$$
\operatorname{Re}=\frac{\rho\left(\frac{\omega d}{2}\right) D}{\mu}
$$


Here $d$ is the diameter of the mixer, $D$ is the diameter of the tank, $\omega$ is the angular velocity of the mixer. The representative velocity and length are the peripheral velocity $\omega d / 2$ and the tank diameter $D$, respectively. The density, $\rho$ is $10^{3}$ $\mathrm{kg} \cdot \mathrm{m}^{-1} \cdot \mathrm{s}^{-1}$ and the viscosity, $\mu$ is $1.0 \times 10^{-3} \mathrm{~Pa} \cdot \mathrm{s}$, at temperature $20^{\circ} \mathrm{C}$.

\subsection{Concentration Measurement}

The experimental apparatus used in concentration measurement is the same as the visualization experiment in Figure 2. The mixer position $h$ was $3 / 4 H$. Initial fluid was separated into two regions by means of buoyancy effect. Figure 3 presents the initial condition of the concentration measurement. The lower half, from 0 to $H / 2$, was filled with usual water with ambient temperature and upper half with blue-dyed water with 5 degrees higher than ambient. The dye is acryl gouache and concentration rate $0.025 \mathrm{~kg} / \mathrm{m}^{3}$. The blue water has been charged carefully to avoid disturbance within enough time. The background of observation was covered with a white board to improve the color diagnostic.

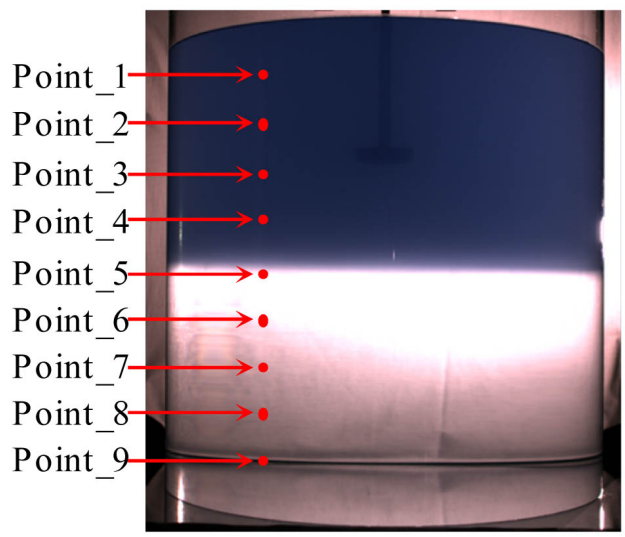

Figure 3. The initial condition of concentration measurement experiment with particular nine vertical points.

For this experiment, a preliminary calibration was carried out to obtain the correlation between the color concentration and image intensity. Figure 4 shows the calibration sampling for six samples out of eleven samples of concentration rates by $10 \%$. Figure 4 (a) shows the image of dyed water, Figure 4 (b) pseudo-color representation of Figure 4 (a) and Figure 4 (c) is the calibration curves measured for specific points. The image intensity was correlated by extracting the saturation of the image of the HSV color model.

As shown in the pseudo-color map, although the correlated image intensity is not uniform due to the refraction and reflection of the source light on the cylindrical surface of the tank and material, we can obtain a local calibration curve between the concentration and the image intensity at any fixed position. Consequently, we can measure the local concentration at any observation point.

Figure 4 (c) presents the local calibration curves described above at the nine points as a sample in Figure 3. The calibrated polynomial curves were obtained 
with the experimental data by means of least squares method. The fitting error, $e$ of the calibration curves has been measured by the root mean square deviation of the image intensity from Figure 4(c) by the following formula

$$
e=\sqrt{\frac{1}{N} \sum_{i=1}^{N}\left(I_{\mathrm{c}}\left(c_{i}\right)-I_{\mathrm{e}}\left(c_{i}\right)\right)^{2}}
$$

here, $N$ is the number of sampling data. $I_{\mathrm{c}}$ and $I_{\mathrm{e}}$ are the image intensity of calibration curve and experimental data, respectively. The deviations of the image intensity, $e$ at point_1 to point_9 are $0.0169,0.0228,0.0175,0.0224,0.0159$, $0.0243,0.0317,0.0084$ and 0.0106 respectively. As shown in Figure 4 (c), for each sample point, one can find a considerable deviation as much as $30 \%$ in low concentration. The error is caused by the reflection and refraction of the source light on the cylindrical surface of the tank. Although the concentration rate is hard to be evaluated with a good accuracy at low concentration rates, we can discuss the mixing process qualitatively in terms of the change of concentration rate.

(a)

(b)

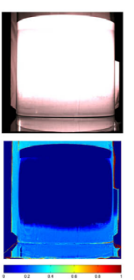

(i) $0 \%$ $\left(0.000 \mathrm{~kg} / \mathrm{m}^{3}\right)$

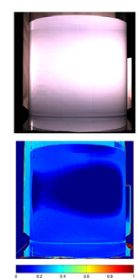

(ii) $20 \%$ $\left(0.005 \mathrm{~kg} / \mathrm{m}^{3}\right)$

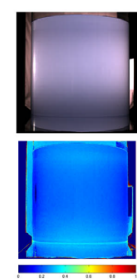

(iii) $40 \%$ $\left(0.010 \mathrm{~kg} / \mathrm{m}^{3}\right)$

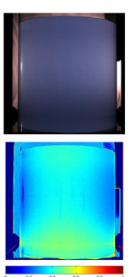

(iv) $60 \%$ $\left(0.015 \mathrm{~kg} / \mathrm{m}^{3}\right)$

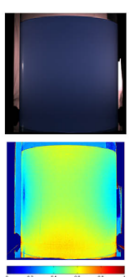

(v) $80 \%$ $\left(0.020 \mathrm{~kg} / \mathrm{m}^{3}\right)$

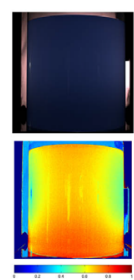

(vi) $100 \%$ $\left(0.025 \mathrm{~kg} / \mathrm{m}^{3}\right)$

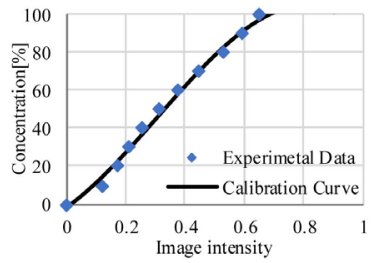

(i) Point_1

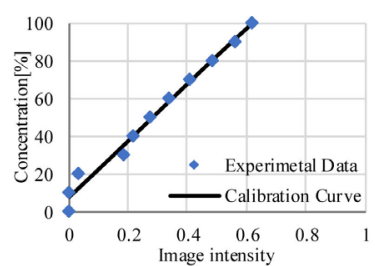

(iv) Point_4

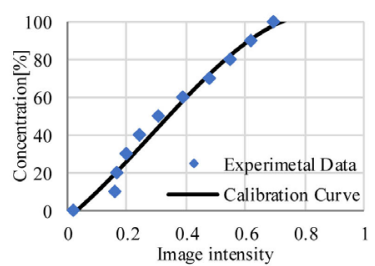

(vii) Point_7

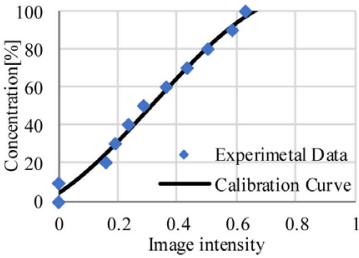

(ii) Point_2

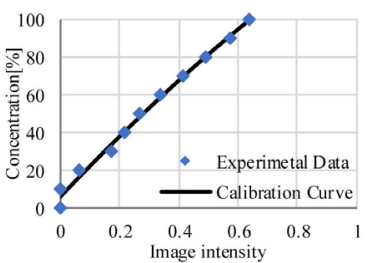

(v) Point_5

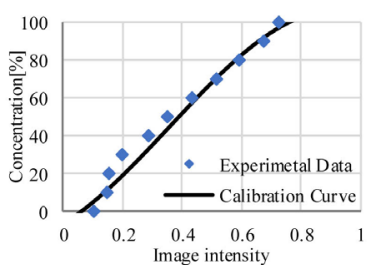

(viii) Point_8

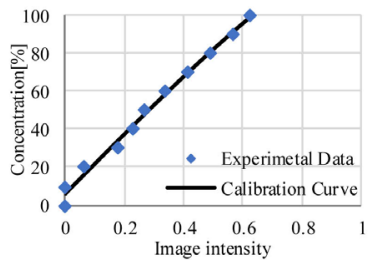

(iii) Point_3

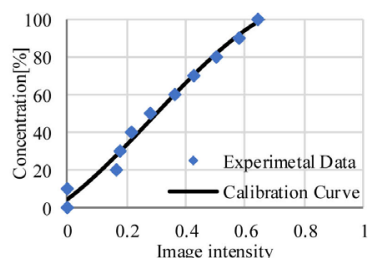

(vi) Point_6

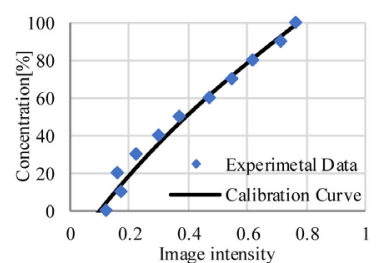

(ix) Point_9

(c)

Figure 4. (a) Dyed water; (b) Image intensity of the dyed water; (c) Local calibration curve of fixed points (positions of points are described in Figure 3). 


\section{Results and Discussion}

\subsection{Visualization Results}

The flow pattern of fin-type and conduit-type mixers have been compared in Figure 5 and Figure 6. By observation of motion pictures, the flow direction was determined by the motion picture, then the red arrows indicate the stream schematically.

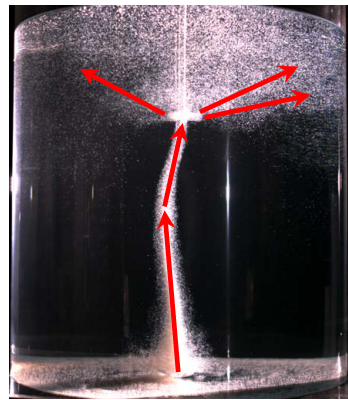

(a)

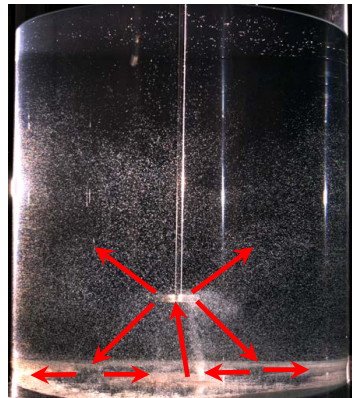

(b)

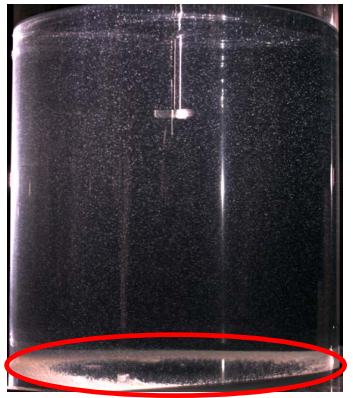

(c)

Figure 5. Flow visualization of fin-type mixer at $n=350 \mathrm{rpm}$. (a) $h=3 / 4 H, t=150 \mathrm{~s}$; (b) $h=1 / 4 H, t=50 \mathrm{~s}$; (c) $h=3 / 4 H, t=15 \mathrm{~min}$.

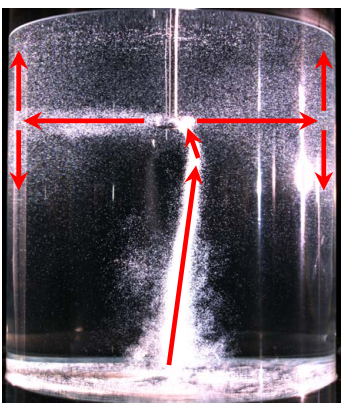

(a)

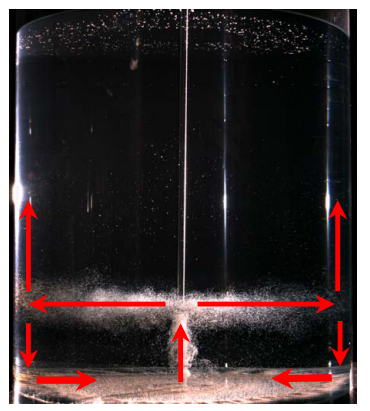

(b)

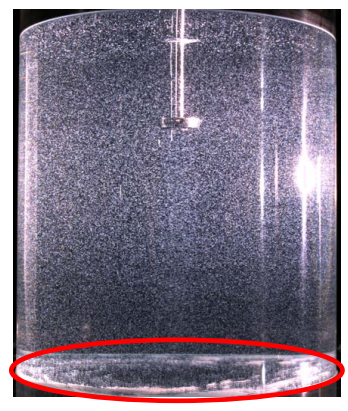

(c)

Figure 6. Flow visualization of conduit-type mixer at $n=350 \mathrm{rpm}$. (a) $h=3 / 4 H, t=150$ s; (b) $h=1 / 4 H, t=40 \mathrm{~s}$; (c) $h=3 / 4 H, t=15 \mathrm{~min}$.

As shown in Figure 5(a) and Figure 5(b), the fin-type mixer generates a suction flow like tornado from the bottom, then, the flow turns towards the radial direction. In the condition in Figure 5(b), the particles are transported from the bottom and scattered to all direction. Also, it was observed that a small fraction of the particles near the bottom was moved towards the corner of the tank. Figure 5(c) shows the suspended tracer pattern after long duration time, $t=15$ min. One can observe a lot of sedimentation on the bottom corner. It was confirmed that the fin-type mixer has poor performance about the stirring at the bottom corner. Though the stirring process of the fin-type mixer near the mixer is strong, the process degenerated at the long distance.

According to Figure 6(a) and Figure 6(b), a strong helical suction was induced by the conduit-type mixer. In this case, spiral flow was generated along a horizontal plane due to the centrifugal force. Near the tank wall, particle trajec- 
tory was divided into upward and downward direction. Figure 6(b), shows that particles can be reached up to the center from the corner of the tank.

After the long-elapsed time, Figure 6(c) shows a small amount of sedimentation at the bottom corner. This fact means that the agitation at the corner of the bottom of the tank by the conduit-type mixer is stronger than that in the fin-type mixer, as shown in Figure 5(c).

Additionally, it was observed that the central vortex and the wave at the upper surface by the fin-type mixer is larger due to the suction flow from the upper surface.

A comparison of flow patterns between the fin-type and the conduit-type based on the flow visualization experiment is illustrated schematically in Figure 7. The fin-type mixer emanates the fluid to the all radial direction. The kinetic motion of emerged fluid was dissipated in short distance. Then we can observe a couple of circulation combining with the two-sided suction, one from the upper region and another one from the lower region, thus, the fin-type mixer generates a closed loop around the mixer.

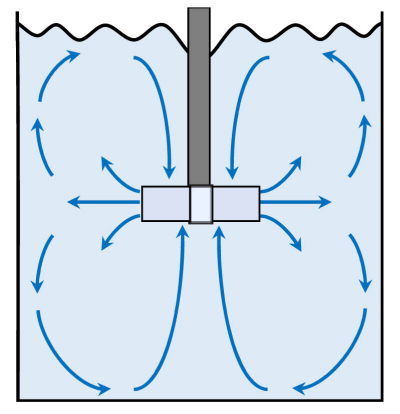

(a)

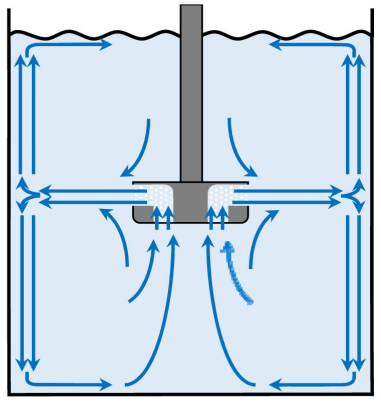

(b)

Figure 7. Schematic diagram of the flow pattern of (a) Fin-type mixer; (b) Conduit-type mixer.

On the other hand, during the rotation of mixer, the nozzles of the conduit-type mixer eject a strong radial jet due to the centrifugal force. Along the horizontal plane, the jets propagated toward the tank wall. On the tank wall, the jets was divided upward and downward along the side wall, which pushes the fluid towards the center from the corner of the tank. From the center of the tank bottom, a strong helical suction pulls the fluid into the mixer and ejects them again as a jet flow. The conduit-type mixer generates a wider and stronger circulatory flow pattern at the lower region of the mixer. Also, the circulation flow in upper region was constructed remarkably (see Figure 6(a) again) but weaker than the fin-type mixer because the central vortex and the wave at the upper surface was weak.

\subsection{Concentration Measurement}

The concentration measurement was carried out to investigate the time sequential progression of local concentration. Here, the characteristic mixing time 
should be determined relative to the equilibrium concentration rate. Mathematically, the mixing time $t_{\mathrm{m}}$ can be estimated to satisfy the following condition as the following,

$$
\frac{\left|c_{i}\left(t_{\mathrm{m}}\right)-c_{i}\left(t_{\infty}\right)\right|}{c_{i}\left(t_{\infty}\right)} \leq \varepsilon \text { for whole field }
$$

here, $c_{i}(t)$ is the concentration at time $t$, and $t_{\infty}$ is the time reaching up to the equilibrium state, $i=1,2, \cdots, 9$ and $\varepsilon=0.05$.

As the sampling proves, 9 equidistant points, were chosen as described in Figure 3 along the vertical line with a radial distance of $D / 4$. Figure 8 presents the results of the concentration experiment. The $x$-axis represents time in seconds and $y$-axis concentration rate. At the initial state, the concentration at the point_5 to point_9 should be $0 \%$, however, some data included an error. This was caused by the reflection and refraction of the source light on the cylindrical surface of the tank as described in Section 2.2.

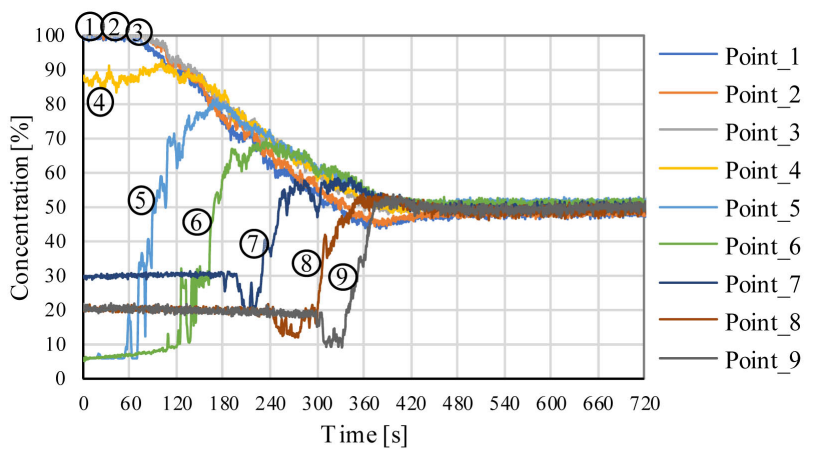

(a)

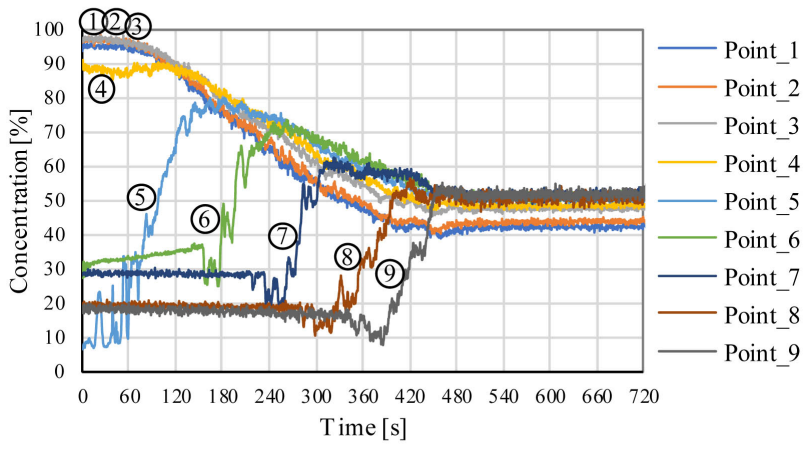

(b)

Figure 8. Concentration rate of points of interest with time (positions are described in Figure 3). (a) Fin type; (b) Conduit type.

The temporal variation of concentration at the 9 points from $t=0$ to $720 \mathrm{~s}$ for fin-type is presented in Figure 8(a). In this figure, the concentration rates at point_1 to point_4 are decreasing after $t=70 \mathrm{~s}$ and those at the point 5 to point_9 increasing at $t=60,130,210,280$, and $330 \mathrm{~s}$, respectively. The concentration rates for the latter points reach the maximum $80 \%, 68 \%, 57 \%, 53 \%$ and $52 \%$ at $t=160,220,270,350$, and $380 \mathrm{~s}$, respectively. All points reached at equi- 
librium concentration till, $t_{\mathrm{m}}=440 \mathrm{~s}$.

Figure 8 (b) presents the concentration at the specific points by the conduit-type mixer. According to this figure, the concentration at point_1 to 4 is decreasing after $t=60 \mathrm{~s}$ and those at point_5 to 9 is increasing at a time approximately, $t=60,180,250,320$, and $390 \mathrm{~s}$, respectively. The concentration rates at latter points reached maximum $78 \%, 70 \%, 60 \%, 55 \%$ and $50 \%$ at $t=180$, $260,320,420$, and $450 \mathrm{~s}$, respectively. All points reach at equilibrium concentration till, $t_{\mathrm{m}}=480 \mathrm{~s}$.

Mixing by conduit-type is slower than by fin-type. This result can be considered as follows. The fin-type mixer generates a closed loop locally near the mixer. So, the exchange rate of the fluid between the upper region and the lower region is faster near the fin-type mixer due to the two-sided suction and closed loop. On the other hand, conduit-type mixer produces a wider circulatory pattern along the tank wall and one-sided suction flow occurred only from the bottom of the tank.

\section{Conclusion}

The flow characteristics of a mixing tank with conduit-type and fin-type mixers were demonstrated by flow visualization and concentration experiment. The conduit-type mixer generates a radial jet stream from the nozzle due to the centrifugal force. This jet flow produces a spiral rotating jet in a horizontal plane. The mixing time of the conduit-type mixer is longer than that of the fin-type mixer because it requires a longer initial time to produce the wider circulatory flow pattern, which is confirmed by concentration measurement and flow visualization. However, the conduit-type mixer provides better uniform mixing than fin-type mixer.

\section{Acknowledgments}

We would like to show our appreciation to Mr. Yushi Koike, Graduate School of Science and Engineering, Saitama University for his great support on our project.

\section{Conflicts of Interest}

The authors declare no conflicts of interest regarding the publication of this paper.

\section{References}

[1] Paul, E.L., Atiemo-Obeng, V.A. and Kresta, S.M. (2004) Handbook of Industrial Mixing Science and Practice. John Wiley \& Sons, Inc., Hoboken.

[2] Harnby, N., Nienow, A.W. and Edwards, M.F. (1997) Mixing in Process Industries. Butterworth-Heinemann, Boston.

[3] Oldshue, J.Y. (1983) Fluid Mixing Technology and Practice. Chemical Engineering, New York

[4] Uhl, V.W. and Gray, J.B. (1966) Mixing: Theory and Practice. Academic Press, 
USA.

[5] Cheremisinoff, N.P. (2000) Handbook of Chemical Processing Equipment. Butterworth-Heinemann, Boston.

[6] Rodriguez, G., Anderlei, T., Micheletti, M., Yianneskis, M. and Ducci, A. (2014) On the Measurement and Scaling of Mixing Time in Orbitally Shaken Bioreactors. Biochemical Engineering Journal, 82, 10-21. https://doi.org/10.1016/j.bej.2013.10.021

[7] Norwood, K.W. and Metzner, A.B. (1960) Flow Patterns and Mixing Rates in Agitated Vessels. AIChE Journal, 6, 432-437. https://doi.org/10.1002/aic.690060317

[8] Lamberto, D.J., Alvarez, M.M. and Muzzio, F.J. (1999) Experimental and Computational Investigation of the Laminar Flow Structure in a Stirred Tank. Chemical Engineering Science, 54, 919-942. https://doi.org/10.1016/S0009-2509(98)00275-9

[9] Hiraoka, S., Kato, Y., Tada, Y., Ozaki, N., Murakami, Y. and Lee, Y.S. (2001) Power Consumption and Mixing Time in an Agitated Vessel with Double Impeller. Chemical Engineering Research and Design, 79, 805-810. https://doi.org/10.1205/02638760152721613

[10] Metzner, A.B. and Taylor, J.S. (1960) Flow Patterns in Agitated Vessels. AIChE Journal, 6, 109-114. https://doi.org/10.1002/aic.690060121

\section{Nomenclature}

c Concentration rate [\%]

d Mixer diameter [m]

$D$ Vessel diameter $[\mathrm{m}]$

e Measurement error [-]

$h \quad$ Mixer position [m]

$H \quad$ Vessel height [m]

I Image intensity [-]

$n \quad$ Rotation rate [rpm]

$t$ Time [s]

$t_{\mathrm{m}}$ Mixing Time [s]

Re Reynolds number

$\mu \quad$ Viscosity [Pa.s]

$\rho \quad$ Density $\left[\mathrm{kg} \cdot \mathrm{m}^{-1} \cdot \mathrm{s}^{-1}\right]$

$\omega$ Angular velocity $\left[\mathrm{s}^{-1}\right]$ 